\title{
HLA-DPB1 Variant Effect on Hepatitis B Virus Clearance and Liver Cirrhosis Development Among Southwest Chinese Population
}

\author{
Zhangyong Hu ${ }^{1, *}$; Jun Yang ${ }^{1}$; Guolian Xiong ${ }^{1}$; Han Shi ${ }^{1}$; Yuan Yuan ${ }^{1}$; Lin Fan ${ }^{1}$; Yali Wang ${ }^{1}$ \\ ${ }^{1}$ Department of Infection Diseases, First Affiliated Hospital, Chengdu Medical College, Chengdu, China \\ ${ }^{*}$ Corresponding Author: Zhangyong Hu, Department of Infection Diseases, First Affiliated Hospital, Chengdu Medical College, Chengdu, China. Tel: +86-15388138508, Fax: +86- \\ 2883016678, E-mail: chendu47@163.com
}

Received: April 30, 2014; Revised: June 26, 2014; Accepted: August 3, 2014

\begin{abstract}
Background: Previous studies have shown that genetic variants in HLA-DP genes affect disease progression in hepatitis B virus (HBV) infection.

Objectives: We aimed to evaluate possible association between HLA-DPB1 rs9277534 polymorphism and different clinical complications of hepatitis B virus (HBV)infection.

Materials and Methods: Snapshot assay was used to investigate the association of rs9277534 polymorphism in 342 patients with persistent $\mathrm{HBV}$ infection and 342 age and gender-matched $\mathrm{HBV}$ spontaneous clearance controls. Patients were categorized into asymptomatic HBV carriers (AsC, $n=104)$, chronic hepatitis $B(C H B, n=116)$, and liver cirrhosis $(L C, n=122$ ) subgroups.

Results: There was a significantly higher proportion of the rs9277534 minor allele A in HBV spontaneous clearance control than that in $\mathrm{HBV}$ persistent infection group $(\mathrm{OR}=0.58,95 \% \mathrm{CI}=0.46-0.73, \mathrm{P}<0.0001)$. Genotypic analysis showed that $\mathrm{GA}$ and AA genotypes were associated with $\mathrm{HBV}$ spontaneous clearance (GA: $\mathrm{OR}=0.56,95 \% \mathrm{CI}=0.40-0.79, \mathrm{P}=0.019 ; \mathrm{AA}: \mathrm{OR}=0.24,95 \% \mathrm{CI}=0.14-0.44, \mathrm{P}<0.0001)$. A significant difference was found between AsC and LC groups in the distribution of AA genotype $(\mathrm{OR}=9.32,95 \% \mathrm{CI}=1.293-67.14, \mathrm{P}=0.027)$. Conclusions:Variant at rs9277534 could affect both the spontaneous clearance of HBV infection and progression from asymptomatic HBV carriers to HBV-related liver cirrhosis in Southwest Han Chinese population.
\end{abstract}

Keywords: Hepatitis B virus; Single Nucleotide Polymorphism; Human; Leukocyte; Antigen

\section{Background}

Hepatitis B virus (HBV) infection results in various clinical complications including HBV spontaneous clearance, asymptomatic HBV carrier (AsC), chronic active hepatitis $\mathrm{B}$ (CHB), fulminant hepatitis, liver cirrhosis (LC), and even hepatocellular carcinoma (HCC) (1). Although the exact mechanisms of HBV persistence are still not fully defined, a complex interplay of viral, environmental, and genetic components including age, gender, viral genotype, host immunity, and infection with other viruses are thought to be associated with persistent HBV infection $(2,3)$. However, host immune response against $\mathrm{HBV}$ is believed to be the most important determinant of different outcomes after HBV infection. Numerous studies suggested that single nucleotide polymorphisms (SNPs) in host gene could correlate with persistence of $\mathrm{HBV}$ infection or HBV spontaneous clearance. A genome-wide association study emphasized the importance of human leukocyte antigen (HLA) SNPs in Japanese and Thai patients with chronic hepatitis $\mathrm{B}$; subsequently, significant associations were reported in other Asian countries (4-10). However, Thomas et al. (11) reported that HLA-DPB1 rs9277534 was significantly associated with HBV recovery in both African-American and European populations; while HLADPB1 rs9277535, which strongly associated with chronic hepatitis B infection in Asia, only had a marginal effect on HBV infection in African-American and European populations. In contrast, the HLA-DPB1 rs9277535 SNP was not associated with HBV infection in Caucasians (12). Though there was a significant association with persistent HBV infection in some studies, results are contradictory regarding the correlation of HLA-DP SNPs and progression of chronic HBV infection $(13,14)$.

\section{Objectives}

The present study involved the selection of rs9277534 within HLA-DPB1 gene to identify the association with different clinical complications after HBV infection in Han Chinese population from Sichuan province of Southwestern China.

\section{Materials and Methods}

\subsection{Ethics Statement}

The research protocol was reviewed and approved by the Ethics Committee of the First Affiliated Hospital of Chengdu Medical College in Sichuan province of Southwestern China. Written informed consent was obtained from all participants, and all samples were blinded. 
Hu Z et al.

\subsection{Study Population}

Three hundred and forty-two unrelated Chinese Han patients with persistent HBV infection were enrolled from the First Affiliated Hospital of Chengdu Medical College. Of which, 104 were chronic asymptomatic HBV carriers (AsC), 116 patients had active chronic hepatitis $\mathrm{B}(\mathrm{CHB})$ infection, and 122 had liver cirrhosis (LC). Persistent HBV infection was defined to have positive results for HBsAg and anti$\mathrm{HBc}$ for at least six months with or without HBeAg positivity. Clinical diagnoses in patients were performed based on the Guideline of Prevention and Treatment for CHB (Chinese Society of Hepatology and Chinese Society of Infectious Diseases, Chinese Medical Association, 2010). A group of 342 age- and sex-matched individuals with spontaneous clearance of HBV (HBV-resolved) enrolled as the control group. HBV-resolved was defined as individuals who spontaneously recovered without treatment and with negative results for HBsAg, $\mathrm{HBeAg}$, and HBV deoxyribonucleic acid (DNA), but positive results for antibody to hepatitis B surface antigen (anti-HBs) and antibody for hepatitis B core antigen (anti-HBc). All individuals were tested to exclude hepatitis C (HCV), hepatitis D (HDV), and human immunodeficiency virus (HIV) infections.

\subsection{Extraction Genomic Deoxyribonucleic Acid and Polymorphisms Genotyping}

Genomic DNA was extracted from peripheral blood leukocytes using TIANamp Blood DNA kit (Qiagen, Hilden, Germany) following the manufacturer's instructions. The concentration and purity of DNA were tested by NanoDrop spectrophotometer (Thermo Fisher Scientific, Wilmington, DE, USA) and DNA was diluted to a final concentration of $10 \mathrm{ng} / \mu \mathrm{L}$. Genotyping was performed by SNaPshot method, described in detail in a previous study (15). The sequence of primers for genotyping was as follow:

rs9277534F: CAAATCAAGTTTAGTGCCCTCA; rS9277534R: GCTGCTGTGCACTGATTGTGTG; rs9277534SR:ttttTTTTTTTTTTTTTTTTTTTTTTTTTTTTTTT TTTTAGTCTGCTCACCATTGAATAGTTAAGAATAG.

Data was analyzed by GeneMapper 4.1 (Applied Biosys- tems, USA). Repeated genotyping in 10\% of randomly selected samples yielded $100 \%$ identical results.

\subsection{Statistical Analysis}

Assumption of Hardy-Weinberg equilibrium was tested for the rs9277534 SNP using DeFinetti program (http:/ ihg.gsf.de/cgi-bin/hw/hwa1.pl). All statistical analyses were conducted using SPSS for Windows (version 18.0; SPSS Inc. Chicago, IL, USA). Chi-square-test was used to compare distribution of genotypes between patients and controls. Odds ratios (ORs) with their 95\% confidence intervals (CIs) were calculated based on the binary logistic regression analysis and were adjusted for age and gender. Pvalue $<0.05$ was considered as statistically significant.

\section{Results}

\subsection{Subjects Characteristic and Hardy-Weinberg Equilibrium}

A total of 342 patients with persistent HBV infection (165 males and 177 females) and 342 age- and sex-matched HBV-resolved controls were enrolled in this study. Baseline characteristics are shown in Table 1. The genotypic distributions of SNP were in accordance with the HardyWeinberg equilibrium in the both groups.

\subsection{Genotypic and Allele Frequencies of rs9277534}

The minor alleles (A allele) of rs9277534 were observed at frequencies of $27.78 \%$ in patients with HBV and $39.77 \%$ in controls, respectively. In the case of G allele as conference, the A allele carriage was significantly associated with HBV spontaneous clearance $(\mathrm{OR}=0.58$; $95 \% \mathrm{CI}$ : 0.46, $0.73 ; \mathrm{P}<0.0001)$. There were significant differences between case and control groups in genotype distributions. Compared to individuals carrying wild-type GG genotype of rs9277534, those with AA or GA genotype had a decreased risk of persistent $\mathrm{HBV}$ infection $(\mathrm{OR}=0.24$; $\mathrm{CI}$ : $0.14,0.44 ; \mathrm{P}<0.0001$ for $\mathrm{AA} ; \mathrm{OR}=0.56$; $\mathrm{CI}: 0.40,0.79 ; \mathrm{P}<$ 0.0001 for GA), indicating a plausible protective role for AX genotype against HBV (Table 2).

\begin{tabular}{|c|c|c|c|c|}
\hline \multirow[t]{2}{*}{ variable } & \multicolumn{3}{|c|}{ Patients $(n=342)$} & \multirow[t]{2}{*}{ Control $(n=342)$} \\
\hline & ASC, $(n=104)$ & $\mathrm{CHB},(\mathrm{n}=116)$ & LC, $(n=122)$ & \\
\hline \multicolumn{5}{|l|}{ Gender } \\
\hline Male & 22 & 51 & 92 & 165 \\
\hline Female & 82 & 65 & 30 & 177 \\
\hline Age, y, Mean \pm SD & $35.5 \pm 12.4$ & $37.0 \pm 14.6$ & $50.6 \pm 11.0$ & $41.6 \pm 14.5$ \\
\hline HBsAg+ & 104 & 116 & 122 & 0 \\
\hline Anti-HBs+ & 0 & 0 & 0 & 289 \\
\hline Anti-HBc+ + & 99 & 107 & 113 & 309 \\
\hline
\end{tabular}

\footnotetext{
a Abbreviations: AsC, Asymptomatic HBV carriers group; CHB, Chronic active hepatitis B group; LC, HBV-related liver cirrhosis group.
} 
Hu Z et al.

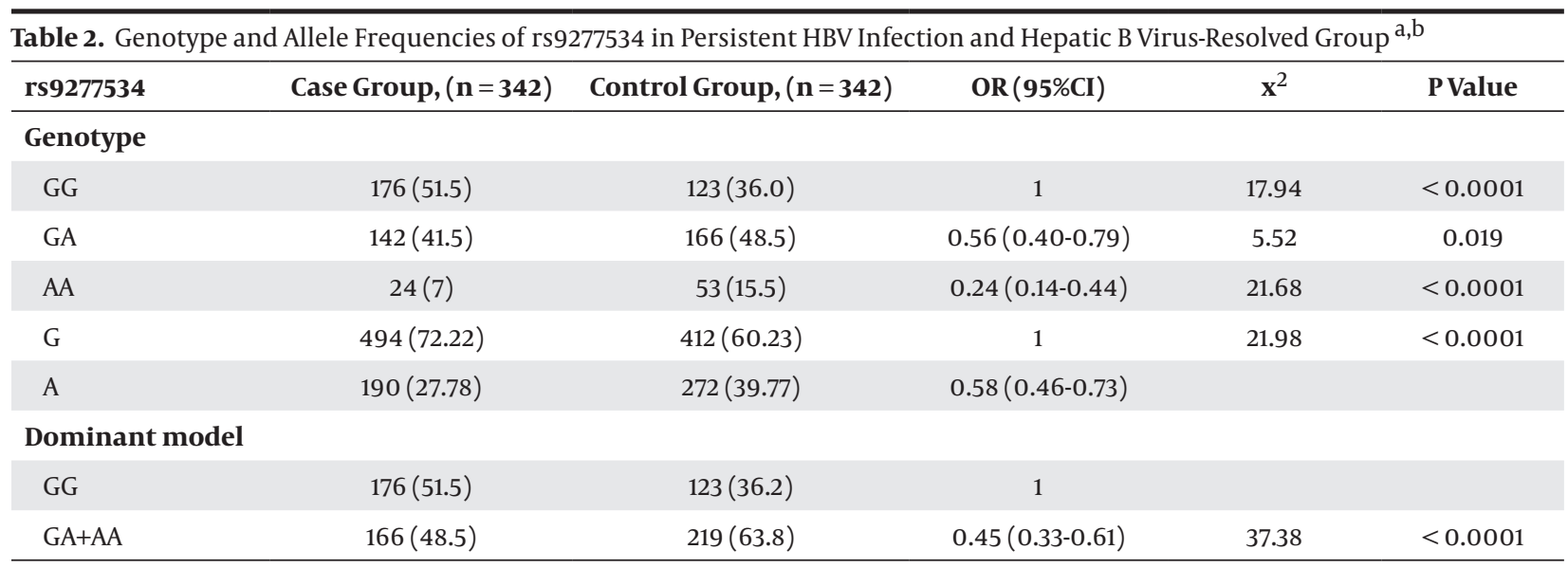

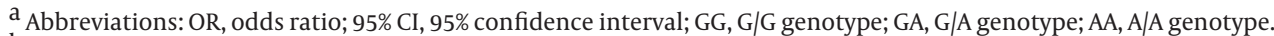

b Case group, persistent HBV infection; control group, HBV-resolved group.

\begin{tabular}{|c|c|c|c|c|}
\hline Genotype & Control, No. (\%) & Case, No. (\%) & Adjusted OR (95\%CI) & P Value \\
\hline \multicolumn{5}{|c|}{ AsC vs. CHB } \\
\hline GG & $58(55.8)$ & $64(55.2)$ & 1 & 0.70 \\
\hline GA & $43(41.3)$ & $47(40.5)$ & $0.99(0.56-1.74)$ & 0.97 \\
\hline AA & $3(2.9)$ & $5(4.3)$ & $1.90(0.41-8.69)$ & 0.41 \\
\hline $\mathrm{GA}+\mathrm{AA}$ & $46(44.2)$ & $52(44.8)$ & $1.02(0.54-1.94)$ & 0.96 \\
\hline \multicolumn{5}{|l|}{ AsC vs. LC } \\
\hline GG & $58(55.8)$ & $54(44.3)$ & 1 & 0.06 \\
\hline GA & $43(41.3)$ & $52(42.6)$ & $0.81(0.36-1.81)$ & 0.61 \\
\hline AA & $3(2.9)$ & $16(13.1)$ & $9.32(1.29-67.14)$ & 0.027 \\
\hline $\mathrm{GA}+\mathrm{AA}$ & $46(44.2)$ & $68(55.7)$ & $1.16(036-3.71)$ & 0.81 \\
\hline \multicolumn{5}{|l|}{ CHB vs. LC } \\
\hline GG & $64(55.2)$ & $54(44.3)$ & 1 & 0.22 \\
\hline GA & $47(40.5)$ & $52(42.6)$ & $1.01(0.52-1.97)$ & 0.98 \\
\hline AA & $5(4.3)$ & $16(13.1)$ & $3.19(0.84-12.08)$ & 0.09 \\
\hline $\mathrm{GA}+\mathrm{AA}$ & $52(44.8)$ & $68(55.7)$ & $1.66(0.58-4.71)$ & 0.34 \\
\hline
\end{tabular}

a Abbreviations: AsC, Asymptomatic HBV carriers; CHB, chronic active Hepatitis B; GG, G/G genotype; GA, G/A genotype; AA, A/A genotype; LC, liver cirrhosis.

\section{3. rs9277534 Polymorphism in Different Persis- tent Hepatic B Virus Infection Subgroups}

To determine whether rs9277534 polymorphism was associated with progression of persistent HBV infection to liver cirrhosis, a similar analysis was performed by comparing LC with AsC group, CHB with AsC group, and LC with CHB group. Unexpectedly, the frequency of rs9277534 AA genotype was significantly higher in LC group than AsC group (OR= 9.32; CI: 1.29, 67.14; $\mathrm{P}=0.027)$, suggesting that patients carriage AA genotype had a higher risk for developing liver cirrhosis (Table 3). However, there were no significant differences between $\mathrm{CHB}$ and $\mathrm{LC}$ groups $(\mathrm{OR}=3.19, \mathrm{CI}: 0.84,12.08 ; \mathrm{P}=0.09)$ after adjustment for age and sex.

\section{Discussion}

Classical human leukocyte antigen loci are classified into two types of class I (HLA-A, B, C, E, F, G, H, and J) and class II (HLA-DR, DP, DQ, DO, and DM) molecules. Following their discovery in 1970s, HLA gene was selected as the major gene to study the susceptibility to infectious diseases (16). HLA-DPA1 and HLA-DPB1 molecules, the central components of MHC class II, are involved in antigen presenting to $\mathrm{CD} 4+\mathrm{T}$ helper cells. Therefore, they are very 
crucial to induce immune response against infectious diseases (17). Accumulating evidences suggested that HLA-DP variants are related to liver diseases. Hirayama et al. (18) confirmed that the HLA-DR-DQ alleles had a protective effect on early changes of liver fibrosis, while the HLADP alleles had a protective role against the late phase of schistosomal hepatic fibrosis. Zhang Q et al. (19) reported that HLA-DP could affect cirrhosis and HCC risks through interacting with HBV mutants. Guo X et al. $(20,21)$ demonstrated that genetic variants in the HLA-DP locus were strongly associated with persistent HBV infection in both Northern and Southern Han Chinese population, but not with HBV progression. O'Brien TR et al. (22) found that rs3077 and rs9277535 were significantly associated with decreased mRNA expression of HLA-DPA1 and HLA-DPB1, respectively. It had been hypothesized that lower expressions of HLA-DPA1 and HLA-DPB1 could be risk predictors of chronic HBV infection. In the present study, there was a significant difference between patients with persistent HBV infection and HBV-resolved controls at both allele and genotype levels, suggesting the rs9277534 minor A allele as a protective factor against HBV infection, which was consistent with the previous study. In addition, stratified analysis by different chronic HBV infection status revealed that there was a significant difference in genotypic frequencies distribution between patients with AsC and LC. Unexpectedly, LC group had a higher frequency of genotype AA than AsC group after age and sex adjustment. Hence, it could be considered that the HLA-DP rs9277534 variant may not only be involved in the process of HBV spontaneous clearance, but it may also be related to the progression of $\mathrm{CHB}$ to end-stage liver disease in Southwest Han Chinese population. Effective $\mathrm{T}$ cell response plays a critical role in HBV clearance, and inadequate HBV-specific cytotoxic T cell (CTL) responses are assumed to be the leading cause of HBV persistent infection (23). Thomas R et al. (11) demonstrated that the rs9277534 GG genotype healthy donors had higher levels of HLADP surface protein in PBMCs and intracellular transcript level expression than individuals carrying non-GG genotypes; perhaps higher HLA-DP expression could promote Th2 dominant immune response along with poor CTL activity in patients with $\mathrm{HBV}$ infection, thus resulting in HBV persistence. While in patients with chronic HBV infection, lower level of HLA-DP protein expression in individuals bearing AA genotype might favor Th1 responses; therefore, on one hand, repeated liver cell injury mediated by inadequate Th1 responses to HBV infection might increase the risk of HBV-related liver cirrhosis and hepatocellular carcinoma. On the other hand, Th1 dominant immune responses associated with polyclonality and multispecificity of HBV-specific CTL responses could completely clear HBV. This assumption might support previous finding revealed by Cheng HR (24) indicating that patients with CHB bearing non-GG genotype of rs9277535, which was reported to be in perfect linkage disequilibrium with SNP rs9277534 in 3'UTR of HLA-DPB1 gene in Chinese population, had higher chance of spontaneous HBsAg seroclearance. In conclusion, the present study showed that carriers with rs9277534 A allele and AA genotype were more likely to clear HBV spontaneously; while in patients with persistent HBV infection, AA genotype could be related to progression of chronic HBV carriers to liver cirrhosis. However, some limitations still exist in this study. Firstly, virus factors such as virus mutants and HBV DNA level were not taken into consideration while evaluating the risk of cirrhosis development. Secondly, a relatively small sample in each subgroup was studied which could lead to insufficient power for statistical analyses. In addition, previous studies concerning the influence of HLA-DP variants on gene expression were conflicting $(11,22)$. Therefore, further studies including a large number of samples are necessary to validate the findings of this study to clarify the potential functions of rs9277534 in HBV infection.

\section{Acknowledgements}

The authors are grateful to the first affiliated hospital of Chengdu Medical college for financial support of this study.

\section{Authors' Contributions}

Zhangyong Hu: Conceived and designed the experiments (50\%), Jun Yang: Conceived and designed the experiments (50\%), Han Shi, Ling Fan, Yuan Yuan, Yali Wang: Performed the experiments, Guolian Xiong: Analyzed the data and revised the manuscript.

\section{Funding/Support}

This study was supported by The First Affiliated Hospital of Chengdu Medical College.

\section{References}

1. Iino S. Natural history of hepatitis B and C virus infections. Oncology. 2002;62 Suppl 1:18-23.

2. Wright TL. Introduction to chronic hepatitis B infection. Am J Gastroenterol. 2006;101 Suppl 1:S1-6.

3. Ganem D, Prince AM. Hepatitis B virus infection--natural history and clinical consequences. N Engl J Med. 2004;350(11):1118-29.

4. Kamatani Y, Wattanapokayakit S, Ochi H, Kawaguchi T, Takahashi A, Hosono N, et al. A genome-wide association study identifies variants in the HLA-DP locus associated with chronic hepatitis B in Asians. Nat Genet. 2009;41(5):591-5.

5. Nishida N, Sawai H, Matsuura K, Sugiyama M, Ahn SH, Park JY et al. Genome-wide association study confirming association of HLA-DP with protection against chronic hepatitis B and viral clearance in Japanese and Korean. PLoS One. 2012;7(6).

6. Mbarek $\mathrm{H}$, Ochi $\mathrm{H}$, Urabe $\mathrm{Y}$, Kumar V, Kubo M, Hosono N, et al. A genome-wide association study of chronic hepatitis B identified novel risk locus in a Japanese population. Hum Mol Genet. 2011;20(19):3884-92.

7. Al-Qahtani AA, Al-Anazi MR, Abdo AA, Sanai FM, Al-Hamoudi W, Alswat KA, et al. Association between HLA variations and chronic hepatitis B virus infection in Saudi Arabian patients. PLoS One. 2014;9(1).

8. Kim YJ, Kim HY, Lee JH, Yu SJ, Yoon JH, Lee HS, et al. A genome-wide association study identified new variants associated with the 
risk of chronic hepatitis B. Hum Mol Genet. 2013;22(20):4233-8.

9. Posuwan N, Payungporn S, Tangkijvanich P, Ogawa S, Murakami S, Iijima S, et al. Genetic association of human leukocyte antigens with chronicity or resolution of hepatitis B infection in thai population. PLoS One. 2014;9(1).

10. Wang L, Wu XP, Zhang W, Zhu DH, Wang Y, Li YP, et al. Evaluation of genetic susceptibility loci for chronic hepatitis B in Chinese: two independent case-control studies. PLoS One. 2011;6(3).

11. Thomas R, Thio CL, Apps R, Qi Y, Gao X, Marti D, et al. A novel variant marking HLA-DP expression levels predicts recovery from hepatitis B virus infection. J Virol. 2012;86(12):6979-85.

12. Vermehren J, Lotsch J, Susser S, Wicker S, Berger A, Zeuzem S, et al. A common HLA-DPA1 variant is associated with hepatitis B virus infection but fails to distinguish active from inactive Caucasian carriers. PLoS One. 2012;7(3)

13. Hu L, Zhai X, Liu J, Chu M, Pan S, Jiang J, et al. Genetic variants in human leukocyte antigen/DP-DQ influence both hepatitis $B$ virus clearance and hepatocellular carcinoma development. Hepatology. 2012;55(5):1426-31.

14. Li J, Yang D, He Y, Wang M, Wen Z, Liu L, et al. Associations of HLADP variants with hepatitis $B$ virus infection in southern and northern Han Chinese populations: a multicenter case-control study. PLoS One. 2011;6(8).

15. Di Cristofaro J, Silvy M, Chiaroni J, Bailly P. Single PCR multiplex SNaPshot reaction for detection of eleven blood group nucleotide polymorphisms: optimization, validation, and one year of routine clinical use. J Mol Diagn. 2010;12(4):453-60.

16. Pieters J. MHC class II-restricted antigen processing and presentation. Adv Immunol. 2000;75:159-208.
17. Handunnetthi L, Ramagopalan SV, Ebers GC, Knight JC. Regulation of major histocompatibility complex class II gene expression, genetic variation and disease. Genes Immun. 2010;11(2):99112.

18. Hirayama K, Chen H, Kikuchi M, Yin T, Gu X, Liu J, et al. HLA-DR-DQ alleles and HLA-DP alleles are independently associated with susceptibility to different stages of post-schistosomal hepatic fibrosis in the Chinese population. Tissue Antigens. 1999;53(3):269-74.

19. Zhang Q, Yin J, Zhang Y, Deng Y, Ji X, Du Y, et al. HLA-DP polymorphisms affect the outcomes of chronic hepatitis B virus infections, possibly through interacting with viral mutations. J Virol. 2013;87(22):12176-86.

20. Guo X, Zhang Y, Li J, Ma J, Wei Z, Tan W, et al. Strong influence of human leukocyte antigen (HLA)-DP gene variants on development of persistent chronic hepatitis B virus carriers in the Han Chinese population. Hepatology. 2011;53(2):422-8.

21. Wong DK, Watanabe T, Tanaka Y, Seto WK, Lee CK, Fung J, et al. Role of HLA-DP polymorphisms on chronicity and disease activity of hepatitis B infection in Southern Chinese. PLoS One. 2013;8(6).

22. O'Brien TR, Kohaar I, Pfeiffer RM, Maeder D, Yeager M, Schadt EE, et al. Risk alleles for chronic hepatitis B are associated with decreased mRNA expression of HLA-DPA1 and HLA-DPB1 in normal human liver. Genes Immun. 2011;12(6):428-33.

23. Webster GJ, Reignat S, Maini MK, Whalley SA, Ogg GS, King A, et al. Incubation phase of acute hepatitis B in man: dynamic of cellular immune mechanisms. Hepatology. 2000;32(5):1117-24.

24. Cheng HR, Liu CJ, Tseng TC, Su TH, Yang HI, Chen CJ, et al. Host genetic factors affecting spontaneous HBsAg seroclearance in chronic hepatitis B patients. PLoS One. 2013;8(1). 